\title{
Observation of Gate-Tunable Coherent Perfect Absorption of Terahertz Radiation in Graphene
}

\author{
Nurbek Kakenov, ${ }^{\dagger}$ Osman Balci, ${ }^{\dagger}$ Taylan Takan, ${ }^{\ddagger}$ Vedat Ali Ozkan, ${ }^{\ddagger}$ Hakan Altan, ${ }^{\ddagger}$ \\ and Coskun Kocabas* ${ }^{*}$,
}

${ }^{\dagger}$ Department of Physics, Bilkent University, 06800 Ankara, Turkey
${ }^{\ddagger}$ Department of Physics, Middle East Technical University, 06800 Ankara, Turkey

Supporting Information

ABSTRACT: We report experimental observation of electrically tunable coherent perfect absorption (CPA) of terahertz $(\mathrm{THz})$ radiation in graphene. We develop a reflection-type tunable $\mathrm{THz}$ cavity formed by a large-area graphene layer, a metallic reflective electrode, and an electrolytic medium in between. Ionic gating in the $\mathrm{THz}$ cavity allows us to tune the Fermi energy of graphene up to $1 \mathrm{eV}$ and to achieve a critical coupling condition at $2.8 \mathrm{THz}$ with absorption of $99 \%$. With the enhanced $\mathrm{THz}$ absorption, we were able to measure the Fermi energy dependence of the transport scattering time of highly doped graphene. Furthermore, we demonstrate flexible active $\mathrm{THz}$ surfaces that yield large modulation in the $\mathrm{THz}$ reflectivity with low insertion losses. We anticipate that the gate-tunable CPA will lead to efficient active $\mathrm{THz}$ optoelectronics applications.

KEYWORDS: graphene, coherent optical absorption, gate-tunable, terahertz, ionic gating, $\mathrm{THz}$ optoelectronics

$\mathrm{T}$ he phenomena of coherent perfect absorption (CPA) is the time-reversed analog of stimulated emission. ${ }^{1-3}$ The optical absorption of a conducting thin film, which is limited to a maximum of $50 \%$ in freestanding form, can be enhanced under illumination of two coherent light beams when they are in-phase on the film. The concept of CPA has been implemented in various materials systems such as metamaterials, ${ }^{4}$ two-level atomic systems, ${ }^{5}$ phase change materials, ${ }^{6}$ plasmonic systems, ${ }^{7}$ and radar-absorbing surfaces. ${ }^{8}$ Very recently the enhancement of optical absorption in twodimensional conductors has attracted great attention for realization of gate-tunable optoelectronic devices. Enhancement of optical absorption in graphene, in particular, plays an important role in broadband tunable optoelectronic devices. The ability to control rates of interband ${ }^{9-11}$ and intraband ${ }^{12}$ electronic transitions via electrostatic gating enables novel active optoelectronic devices. At optical wavelengths, the optical absorption in graphene is limited to $2.3 \%$; $^{10,11,13}$ however for longer wavelengths $\left(\mathrm{THz}^{12,14,15}\right.$ and microwave ${ }^{8}$ ) absorption can be increased up to $50 \%$ when the surface impedance of graphene $\left(Z_{\mathrm{G}}\right)$ matches half of the free space impedance, ${ }^{16} Z_{\mathrm{G}}=1 / \sigma(\omega)=Z_{0} / 2$ where $Z_{0}$ is the free space impedance and $\sigma(\omega)$ is the optical conductivity (see the small signal model given in Supporting Information Figure S1). To enhance the optical absorption further, various device structures have been explored. Patterning graphene into ribbons leads to enhanced absorption due to the localized plasmon oscillations. Fang et al. demonstrated absorption of $20 \%$ in far-IR frequencies. ${ }^{17}$ Placing graphene on a photonic crystal cavity ${ }^{18}$ or inside a microcavity ${ }^{19,20}$ enhances the absorption due to multiple passes. Very recently, Thareja et al. placed graphene at a quarter-wave distance from a metallic surface and showed enhancement up to $5.5 \%$ in IR wavelengths. ${ }^{21-23}$ With the help of local plasma frequency, complete optical absorption at IR frequencies has been proposed using periodically patterned doped graphene. ${ }^{24,25}$

Gate-tunable coherent absorption in graphene at terahertz frequencies has more technological importance because of being a low-cost alternative material for active $\mathrm{THz}$ devices. The recent theoretical studies show that gating graphene near a reflective surface would yield gate-tunable CPA for terahertz radiation. ${ }^{26,27}$ They predicted that, under coherent illumination, $100 \%$ of $\mathrm{THz}$ radiation can be absorbed by a highly doped monolayer graphene when the Fermi energy is close to $1 \mathrm{eV}$. Varying the doping level, $\mathrm{THz}$ absorption can be controlled efficiently by electrical means. This is a challenging requirement. Although the static CPA in graphene for microwave ${ }^{28}$ and visible ${ }^{29,30}$ spectra has been reported, due to the limitation of conventional gating schemes, the gate-tunable $\mathrm{CPA}$ of $\mathrm{THz}$ radiation in graphene has not been observed yet. ${ }^{12,31}$ In our previous works, we used ionic gating to control optical properties of graphene in a very broad spectrum extending from visible to microwave wavelengths. ${ }^{8,13,14,32}$ In this Letter, we demonstrate a new type of tunable $\mathrm{THz}$ cavity that enables us to observe gate-tunable CPA. Figure 1a shows a schematic drawing of our device structure. The large-area monolayer

Received: April 6, 2016

Published: July 25, 2016 


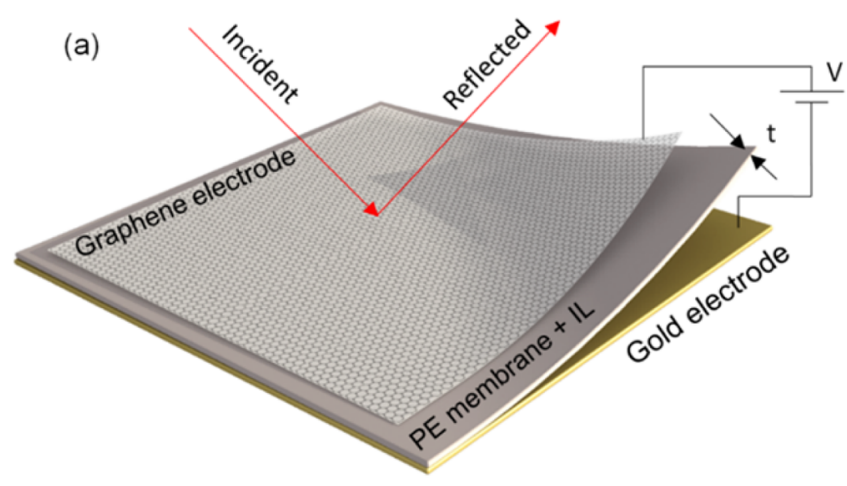

(b)

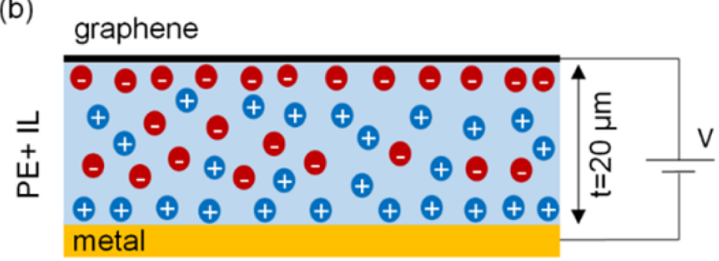

Figure 1. Active $\mathrm{THz}$ surfaces. (a) Schematic representation of electrically tunable $\mathrm{THz}$ cavity used for the coherent perfect absorption in graphene. The $\mathrm{THz}$ cavity is formed by a porous membrane sandwiched between graphene and gold electrodes. The thickness of the membrane is $20 \mu \mathrm{m}$. The ionic liquid electrolyte is soaked into the membrane. (b) Cross-sectional view of the cavity showing the formation of electrical double layers on the graphene and gold electrodes.

graphene is synthesized by chemical vapor deposition on copper foils and then transferred onto a $20-\mu \mathrm{m}$-thick porous polyethylene membrane ( $42 \%$ porosity) that is placed on a reflective gold electrode. The thickness of the membrane defines the cavity length and the resonance wavelength. The gold electrode operates both as the back-reflecting mirror and the gate electrode. We soaked the membrane with roomtemperature ionic liquid (diethylmethyl(2-methoxyethyl)ammonium bis(trifluoromethylsulfonyl)imide, [deme][Tf2N]), which has a large electrochemical window that yields tunable Fermi energy on graphene up to $1 \mathrm{eV}$. Both the electrolyte and PE membrane are transparent between 0.1 and $15 \mathrm{THz}$ (Supporting Information Figure S2). Figure $1 \mathrm{~b}$ shows a schematic cross-sectional view of the device under a bias voltage that polarizes the ionic liquid in the membrane and forms electrical double layers (EDLs) near the graphene and gold interface. The EDL electrostatically dopes the graphene layer and alters its conductivity. Since the thickness of the EDL is very thin for ionic liquids, this configuration yields very large electric field and induced charges on the surface. The advantage of this device is that it provides a very efficient gating scheme with a charge density up to $10^{14} \mathrm{~cm}^{-2}$ and Fermi energy of $1 \mathrm{eV}$ of the open graphene surface. These doping levels are enough to satisfy the CPA condition at $\mathrm{THz}$ frequencies. Our device yields a single-channel CPA when the incident and reflected $\mathrm{THz}$ beams are in phase at the graphene interface. For our device structure, the resonance condition can be written as $t$ $\cos (\theta)=(2 m+1) \lambda / 4 n$ where $\theta$ is the incidence angle, $t$ is the thickness of the membrane, $m$ is an integer, and $n$ is the index of refraction of the cavity. Spectroscopic measurements provide the resonances and antiresonances, which yield perfect and no absorption conditions, respectively.
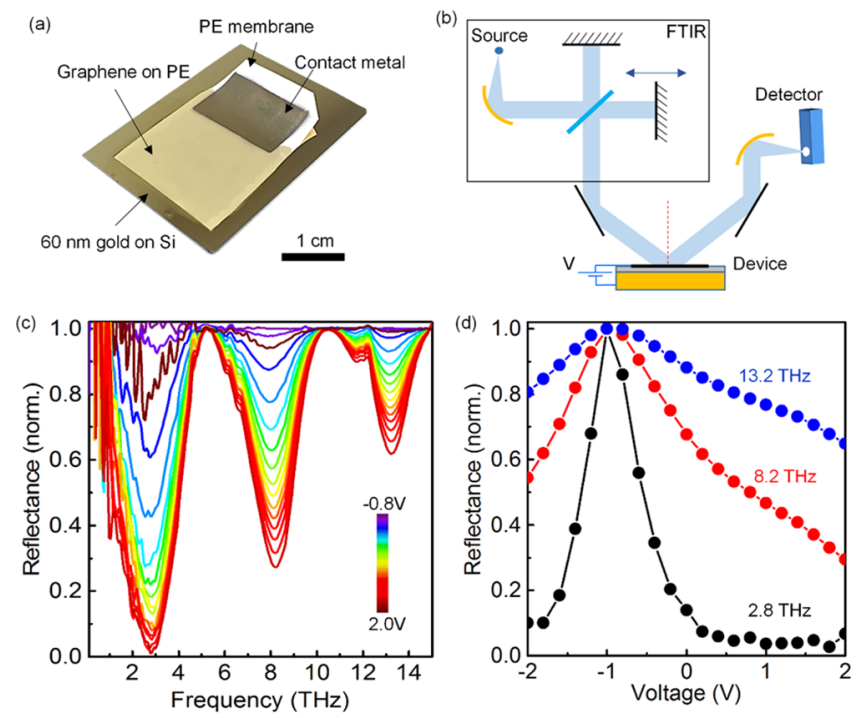

Figure 2. Coherent perfect absorption of $\mathrm{THz}$ radiation. (a) Photograph of the fabricated $\mathrm{THz}$ cavity. The monolayer graphene is transferred onto a PE membrane and placed on a gold-coated substrate. The $20-\mu \mathrm{m}$-thick membrane defines the cavity length, holds the electrolyte, and forms the mechanical support for graphene. (b) Experimental setup used for the $\mathrm{THz}$ measurements. (c) Reflectivity spectrum from the device at different bias voltages. (d) Variation of the resonance reflectance with gate voltage. The charge neutrality point is at $-1 \mathrm{~V}$.

Figure 2a shows the fabricated device. We measured $\mathrm{THz}$ reflection from the biased device using a Fourier transform infrared spectrometer (FTIR) equipped with a far-IR detector and a far-IR source (Figure $2 b$ ). Since ionic liquids have very low vapor pressure, we recorded the reflection spectrum under a vacuum (10 mTorr) to remove the absorption of water. Figure $2 c$ shows the measured reflectivity spectrum from the device under different bias voltages. For a membrane thickness of $20 \mu \mathrm{m}$ and incidence angle of $30^{\circ}$, we observed multiple resonance absorptions at 2.83, 8.24, and $13.23 \mathrm{THz}$ frequencies. For the first resonance, we obtained an absorption of $99 \%$ at $2.0 \mathrm{~V}$ bias voltage. Unlike the condition of the freestanding film, CPA occurs when the real part of the optical conductivity of doped graphene reaches the values $\sigma(\omega)=1 / Z_{0}$ where $Z_{0}$ is the free space impedance. The optical conductivity of graphene at $\mathrm{THz}$ frequencies can be described with the Drude response as

$$
\sigma(\omega)=\frac{e^{2}}{\pi \hbar} \frac{i E_{\mathrm{F}}}{\omega+i \tau^{-1}}
$$

where $E_{\mathrm{F}}$ is the Fermi energy and $\tau$ is the transport scattering time. For high doping levels, $\tau$ varies with the Fermi energy. We observed perfect absorption at low $\mathrm{THz}$ frequencies $(<5 \mathrm{THz})$. For higher frequencies, however, the required doping levels exceed the accessible levels with the present device. The variation of the resonance reflectivity of the first three resonances is plotted in Figure $2 \mathrm{~d}$ against the bias voltage. The reflectivity is normalized by the reflection at the charge neutrality point (CNP, around $-1 \mathrm{~V})$. The large shift in the $\mathrm{CNP}$ is associated with the work function difference between the graphene and gold electrodes. We obtained $99 \%, 76 \%$, and $42 \%$ absorption for $2.83,8.24$, and $13.23 \mathrm{THz}$ frequencies, respectively. To observe perfect absorption for higher order modes, we need larger voltages that exceed the electrochemical 
window of the electrolyte and introduce irreversible damage to the graphene electrode.

The Fermi energy provides a wealth of information about the electrical and optical properties of the device. Liu et al. predicted that, to achieve $\mathrm{CPA}$ in $\mathrm{THz}$ frequencies, the Fermi energy of graphene should be close to $1 \mathrm{eV}$, which yields the required optical conductance for the critical coupling. Near-IR and IR (see Supporting Information Figure S4) reflection spectra from the device provide direct measurement of the Fermi energy of the doped graphene. Figure 3a shows the
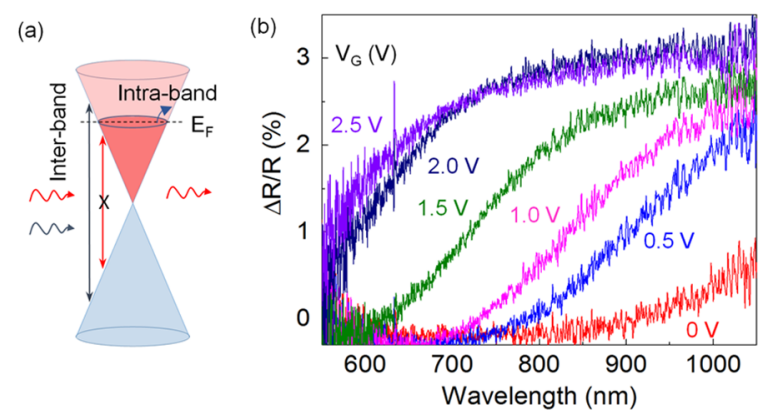

(c)
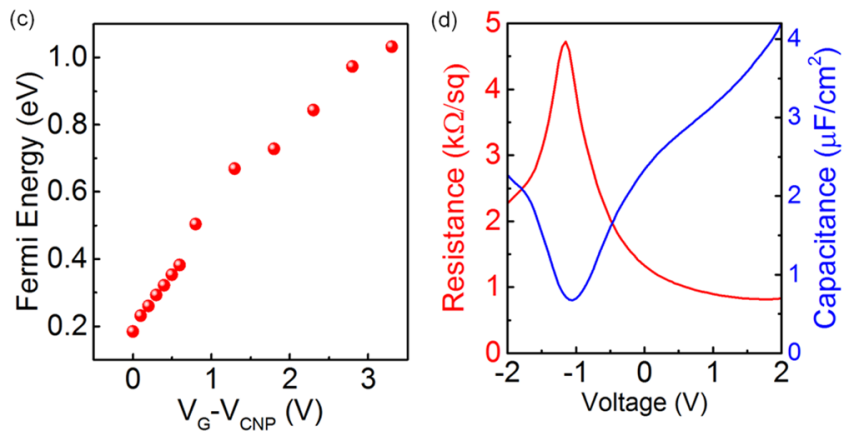

Figure 3. Electrical and optical characterization of the device. (a) Schematic representation of the band structure of graphene and possible electronic transitions. (b) Gate-tunable near-IR optical reflection from the graphene surface at different bias voltages. The number on the curves shows the bias voltage. (c) Fermi energy extracted from the reflection spectrum. (d) Variation of the resistance and capacitance of the devices with the bias voltage. At the charge neutrality point, resistance reaches a maxima of $4.5 \mathrm{k} \Omega$ and the capacitance goes to a minima of $0.8 \mu \mathrm{F} / \mathrm{cm}^{2}$.

electronic band structure of doped graphene. Due to Pauli blocking, doped graphene has a gap in the optical absorption for photon energies $E<2 E_{\mathrm{F}}$. Gating graphene results in an increase in the absorption gap and a step-like change in the reflectivity spectrum. Figure $3 \mathrm{~b}$ shows the measured reflectivity spectra, which show a step-like change in the reflectivity with a cutoff wavelength at $2 E_{\mathrm{F}}$. Although monolayer graphene absorbs around $1.8 \%$ on a dielectric substrate, in our cavity structure, the reflectivity shows about $3 \%$ modulation due to multiple passes. Figure $3 \mathrm{c}$ shows the extracted Fermi energy as a function of bias voltage. At the charge neutrality point $\left(V_{\mathrm{CNP}}\right.$ $=-1 \mathrm{~V}$ ) the unintentional doping level is $0.2 \mathrm{eV}$ and increases linearly with a gate voltage up to $1 \mathrm{eV}$. At $V_{\mathrm{G}}=0 \mathrm{~V}$, graphene is significantly doped with a Fermi energy of $0.55 \mathrm{eV}$ due to the work function difference between the gold and graphene electrodes. To get more insight, we performed electrical characterization of the device using an LRC meter. Figure $3 \mathrm{~d}$ shows the variation of the resistance and capacitance of the devices with the bias voltage. At the charge neutrality point, the sheet resistance reaches $4.5 \mathrm{k} \Omega$ and decreases down to $0.8 \mathrm{k} \Omega$, which also includes the contact resistance of the electrodes. The capacitance of the device shows a minima $\left(0.8 \mu \mathrm{F} / \mathrm{cm}^{2}\right)$ at the charge neutrality point due to the minimum quantum capacitance of the graphene layer. The electrical characterization shows a good agreement with the spectroscopic measurements. Our results suggest that the critical coupling condition is achieved when the Fermi energy is around $1 \mathrm{eV}$.

The thickness of the porous substrates and the incidence angle define the frequency of the resonance absorption. We repeat our measurements with different membrane thicknesses. Figure 4 shows the gate-tunable reflectivity spectrum from two

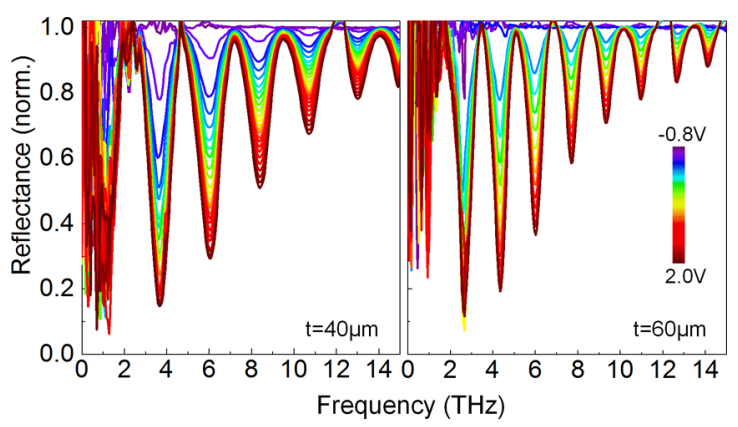

Figure 4. Tunable reflectivity from various $\mathrm{THz}$ cavities: Reflectance spectrum of $\mathrm{THz}$ cavities with 40 and $60 \mu \mathrm{m}$ membrane thickness.

different devices with 40 and $60 \mu \mathrm{m}$ cavity lengths. The observed resonance wavelengths satisfy the critical coupling condition as $\lambda_{\mathrm{m}}=4 n t \cos (\theta) /(2 m+1)$. We do not observe a significant change in the frequency; however, the width of the resonance varies slightly with the bias voltage (see Supporting Information Figure S4). The fundamental resonances of the large cavities are buried under the noise level, due to the sensitivity of the FTIR system at low frequencies $(<2 \mathrm{THz})$. We performed additional experiments using continuous wave tunable frequency $\mathrm{THz}$ sources (see Supporting Information Figure S5). Similarly, we obtained $98 \%$ modulation at 0.368 THz.

Recently, several $\mathrm{THz}$ pump-probe studies revealed semiconducting-to-metallic photoconductivity crossover in doped graphene. ${ }^{33-35}$ These observations are due to the changes of the Drude weight and transport scattering time by the doping level. The enhanced optical absorption of graphene in the tunable $\mathrm{THz}$ cavity could provide a new platform to elucidate nonideal Drude responses of graphene at high doping levels. Due to the frequency dependence of the optical conductivity, the maximum absorbance decreases with frequency. By combining this frequency dependence with the direct measurement of the Fermi energy, we can extract the transport scattering time and its dependence on the Fermi energy. In Figure $5 \mathrm{a}$, we plot the normalized resonance absorbance, which is proportional to the real part of the normalized optical conductivity, $\sigma(\omega) / \sigma_{\mathrm{DC}}=i /\left(\omega+i \tau^{-1}\right)$, against the frequency for varying the Fermi energy between 0.3 and $1.1 \mathrm{eV}$. Although at low doping concentration the $\mathrm{THz}$ response of graphene can be modeled with a Drude model with constant scattering time, at high doping levels, however, the scattering rate changes with the Fermi energy. The transport scattering time has two contributions associated with the long-range charge impurity scattering $\left(\tau_{\mathrm{c}}\right)$ and short-range disorder scattering $\left(\tau_{\mathrm{s}}\right)$ as $\tau^{-1}=$ $\tau_{\mathrm{c}}^{-1}+\tau_{\mathrm{s}}^{-1}$. $^{26,36}$ These scattering mechanisms scale differently with the Fermi energy. For short-range scattering, the scattering rate is proportional to $E_{\mathrm{F}}$; however, for long-range scattering, 

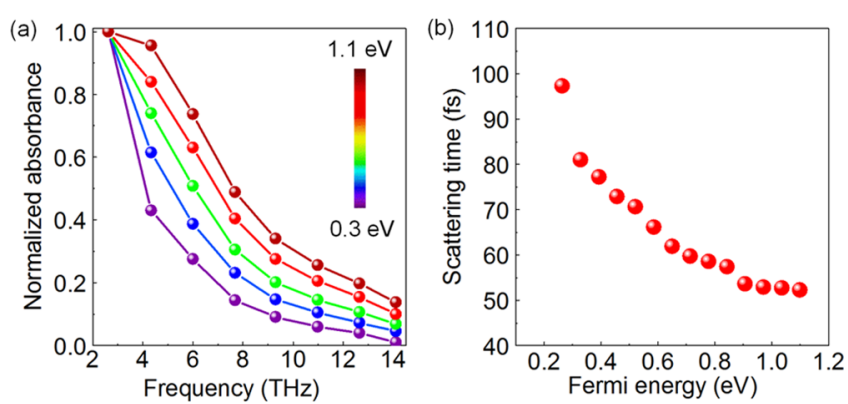

Figure 5. Drude response of graphene at high doping level. (a) Frequency dependence of the resonance absorbance at different Fermi energies. The absorbance is normalized by maximum absorbance at 2.8 THz. (b) Variation of the transport scattering time with the Fermi energy.

the scattering rate is inversely proportional to $E_{\mathrm{F}}$. Our results show that, as the Fermi energy increases, the absorbance decays slower with increasing frequency, indicating a smaller scattering time. Using the Drude model, we extracted the Fermi energy dependence of the total scattering time (Figure 5b). Around the charge neutrality point, the scattering time is close to $100 \mathrm{fs}$ and decreases down to $50 \mathrm{fs}$ at Fermi energies of $1.1 \mathrm{eV}$.

To show the promises of our approach, we demonstrate flexible active $\mathrm{THz}$ surfaces as the application part of our work. The tunable coherent absorption of $\mathrm{THz}$ radiation can lead to new types of active $\mathrm{THz}$ devices. Conventional $\mathrm{THz}$ devices are rigid, which prevents realization of flexible $\mathrm{THz}$ components. The atomic thickness of graphene together with the simple device geometry allows us to fabricate a tunable $\mathrm{THz}$ cavity on a flexible polymer substrate. Figure 6a shows a photograph of
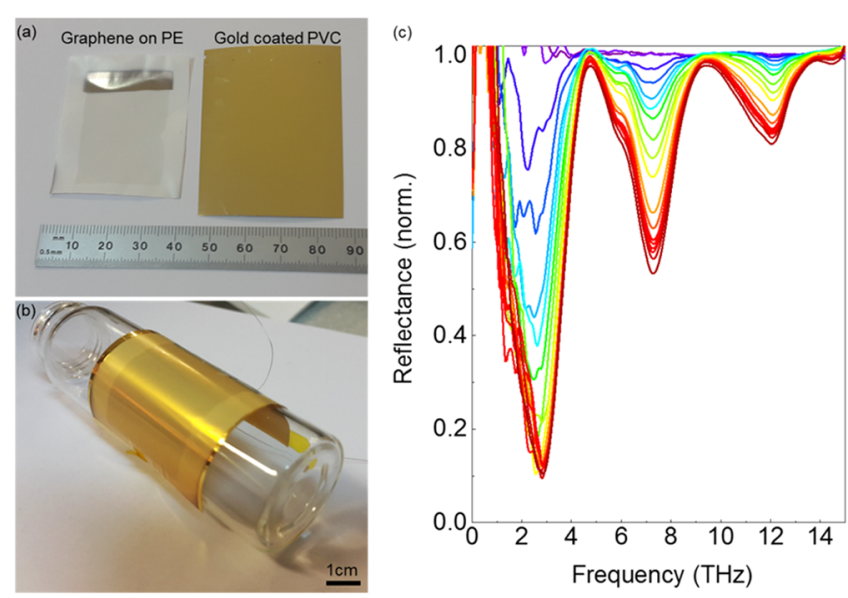

Figure 6. Flexible active $\mathrm{THz}$ surfaces. (a) Photograph of the largearea graphene on PE membrane and gold-coated PVC substrate. (b) Fabricated device rolled around a glass cylinder with a diameter of 2.7 $\mathrm{cm}$. (c) $\mathrm{THz}$ reflectivity spectrum from the curved surface at different bias voltages.

large-area graphene $\left(2.5 \times 3.0 \mathrm{~cm}^{2}\right)$ on a porous $\mathrm{PE}$ membrane and gold-coated PVC substrate. After injecting an ionic liquid into the PE membrane ( $20 \mu \mathrm{m}$ thick), we placed it on the goldcoated PVC substrate and rolled the device around a glass cylinder with a diameter of $2.7 \mathrm{~cm}$ (Figure 6b). We measured the variation of the $\mathrm{THz}$ reflectivity from the curved surface. During the measurement the beam size is set to $6 \mathrm{~mm}$ in diameter. Similar to the rigid devices, we observed three resonances, at 3, 7.2, and $12.2 \mathrm{THz}$ (Figure 6c). The first resonance yields gate-tunable absorption up to $95 \%$ at $2 \mathrm{~V}$ bias voltage.

In conclusion, we report experimental observation of gatetunable coherent perfect absorption of terahertz radiation in highly doped graphene. Our work has four novel parts. First, we developed an electrically tunable $\mathrm{THz}$ cavity using a $\mathrm{THz}$ transparent porous membrane soaked with an ionic liquid electrolyte sandwiched between graphene and gold electrodes. In this device geometry the gold electrode operates as both reflecting mirror and the gate electrode. Second, we observed the coherent perfect absorption of $\mathrm{THz}$ radiation in graphene. The ability to gate graphene up to $1 \mathrm{eV}$ Fermi levels in the $\mathrm{THz}$ cavity allows us to observe a critical coupling condition that yields an absorption of $99 \%$. Third, this novel device configuration allows direct measurement of the Fermi energy and elucidating the doping dependence of the transport scattering time, which varies from $100 \mathrm{fs}$ down to $50 \mathrm{fs}$ as the Fermi energy changes from 0.2 to $1.1 \mathrm{eV}$. Finally, using these structures we demonstrated flexible active $\mathrm{THz}$ surfaces with voltage-controlled $\mathrm{THz}$ reflectance. We anticipate that our work providing a developed device structure as a new platform to study gate-tunable CPA will lead to efficient active $\mathrm{THz}$ components such as tunable $\mathrm{THz}$ mirrors and modulators.

\section{ASSOCIATED CONTENT}

\section{S Supporting Information}

The Supporting Information is available free of charge on the ACS Publications website at DOI: 10.1021/acsphotonics.6b00240.

Transmission line models for the freestanding graphene and the active device, $\mathrm{THz}$ transmittance of the $\mathrm{PE}$ membrane, voltage-controlled IR reflectivity from the device, variation of the center frequency and width of the resonance with the bias voltage, voltage-controlled reflectivity of the device characterized by $0.368 \mathrm{THz}$ continuous $\mathrm{THz}$ source (PDF)

\section{AUTHOR INFORMATION}

\section{Corresponding Author}

*E-mail: ckocabas@fen.bilkent.edu.tr.

\section{Notes}

The authors declare no competing financial interest.

\section{ACKNOWLEDGMENTS}

This work was partially supported by the Scientific and Technological Research Council of Turkey (TUBITAK) grant no. 114F379 and the European Research Council (ERC) Consolidator Grant ERC-682723 SmartGraphene. N.K. acknowledges the TUBITAK-BIDEB 2215 scholarship program.

\section{REFERENCES}

(1) Chong, Y. D.; Ge, L.; Cao, H.; Stone, A. D. Coherent Perfect Absorbers: Time-Reversed Lasers. Phys. Rev. Lett. 2010, 105, 053901.

(2) Wan, W. Time-reversed lasing and interferometric control of absorption (vol 331, pg 889, 2011). Science 2011, 334, 1058-1058.

(3) Pu, M. B.; Feng, Q.; Wang, M.; Hu, C. G.; Huang, C.; Ma, X. L.; Zhao, Z. Y.; Wang, C. T.; Luo, X. G. Ultrathin broadband nearly perfect absorber with symmetrical coherent illumination. Opt. Express 2012, 20, 2246-2254.

(4) Feng, S.; Halterman, K. Coherent perfect absorption in epsilonnear-zero metamaterials. Phys. Rev. B: Condens. Matter Mater. Phys. 2012, 86, 165103. 
(5) Longhi, S. Coherent perfect absorption in a homogeneously broadened two-level medium. Phys. Rev. A: At., Mol., Opt. Phys. 2011, 83, 055804.

(6) Kats, M. A.; Sharma, D.; Lin, J.; Genevet, P.; Blanchard, R.; Yang, Z.; Qazilbash, M. M.; Basov, D. N.; Ramanathan, S.; Capasso, F. Ultrathin perfect absorber employing a tunable phase change material. Appl. Phys. Lett. 2012, 101, 221101.

(7) Noh, H.; Chong, Y. D.; Stone, A. D.; Cao, H. Perfect coupling of light to surface plasmons by coherent absorption. Phys. Rev. Lett. 2012, $108,186805$.

(8) Balci, O.; Polat, E. O.; Kakenov, N.; Kocabas, C. Grapheneenabled electrically switchable radar-absorbing surfaces. Nat. Commun. 2015, 6, 6628.

(9) Wang, F.; Chen, C. F.; Park, C. H.; Boudouris, B. W.; Horng, J.; Geng, B. S.; Girit, C.; Zettl, A.; Crommie, M. F.; Segalman, R. A.; Louie, S. G. Controlling inelastic light scattering quantum pathways in graphene. Nature 2011, 471, 617-620.

(10) Zhang, X.; Liu, M.; Yin, X. B.; Ulin-Avila, E.; Geng, B. S.; Zentgraf, T.; Ju, L.; Wang, F. A graphene-based broadband optical modulator. Nature 2011, 474, 64-67.

(11) Wang, F.; Zhang, Y. B.; Tian, C. S.; Girit, C.; Zettl, A.; Crommie, M.; Shen, Y. R. Gate-variable optical transitions in graphene. Science 2008, 320, 206-209.

(12) Sensale-Rodriguez, B.; Yan, R. S.; Kelly, M. M.; Fang, T.; Tahy, K.; Hwang, W. S.; Jena, D.; Liu, L.; Xing, H. G. Broadband graphene terahertz modulators enabled by intraband transitions. Nat. Commun. 2012, 3, 780.

(13) Polat, E. O.; Kocabas, C. Broadband Optical Modulators Based on Graphene Supercapacitors. Nano Lett. 2013, 13, 5851-5857.

(14) Kakenov, N.; Balci, O.; Polat, E. O.; Altan, H.; Kocabas, C. Broadband terahertz modulators using self-gated graphene capacitors. J. Opt. Soc. Am. B 2015, 32, 1861-1866.

(15) Kakenov, N.; Takan, T.; Ozkan, V. A.; Balci, O.; Polat, E. O.; Altan, H.; Kocabas, C. Graphene-enabled electrically controlled terahertz spatial light modulators. Opt. Lett. 2015, 40, 1984-1987.

(16) Bosman, H.; Lau, Y. Y.; Gilgenbach, R. M. Microwave absorption on a thin film. Appl. Phys. Lett. 2003, 82, 1353-1355.

(17) Fang, Z. Y.; Wang, Y. M.; Schather, A. E.; Liu, Z.; Ajayan, P. M.; de Abajo, F. J. G.; Nordlander, P.; Zhu, X.; Halas, N. J. Active Tunable Absorption Enhancement with Graphene Nanodisk Arrays. Nano Lett. 2014, 14, 299-304.

(18) Majumdar, A.; Kim, J.; Vuckovic, J.; Wang, F. Electrical Control of Silicon Photonic Crystal Cavity by Graphene. Nano Lett. 2013, 13, 515-518.

(19) Engel, M.; Steiner, M.; Lombardo, A.; Ferrari, A. C.; Lohneysen, H. V.; Avouris, P.; Krupke, R. Light-matter interaction in a microcavity-controlled graphene transistor. Nat. Commun. 2012, 3, 906.

(20) Furchi, M.; Urich, A.; Pospischil, A.; Lilley, G.; Unterrainer, K.; Detz, H.; Klang, P.; Andrews, A. M.; Schrenk, W.; Strasser, G.; Mueller, T. Microcavity-Integrated Graphene Photodetector. Nano Lett. 2012, 12, 2773-2777.

(21) Thareja, V.; Kang, J. H.; Yuan, H. T.; Milaninia, K. M.; Hwang, H. Y.; Cui, Y.; Kik, P. G.; Brongersma, M. L. Electrically Tunable Coherent Optical Absorption in Graphene with Ion Gel. Nano Lett. 2015, 15, 1570-1576.

(22) Liu, Y. H.; Chadha, A.; Zhao, D. Y.; Piper, J. R.; Jia, Y. C.; Shuai, Y. C.; Menon, L.; Yang, H. J.; Ma, Z. Q.; Fan, S. H.; Xia, F. N.; Zhou, W. D. Approaching total absorption at near infrared in a large area monolayer graphene by critical coupling. Appl. Phys. Lett. 2014, 105, 181105.

(23) Woo, J. M.; Kim, M. S.; Kim, H. W.; Jang, J. H. Graphene based salisbury screen for terahertz absorber. Appl. Phys. Lett. 2014, 104, 081106.

(24) Thongrattanasiri, S.; Koppens, F. H. L.; de Abajo, F. J. G. Complete Optical Absorption in Periodically Patterned Graphene. Phys. Rev. Lett. 2012, 108, 047401.
(25) Zhang, J. F.; Guo, C. C.; Liu, K.; Zhu, Z. H.; Ye, W. M.; Yuan, X. D.; Qin, S. Q. Coherent perfect absorption and transparency in a nanostructured graphene film. Opt. Express 2014, 22, 12524-12532.

(26) Liu, F. L.; Chong, Y. D.; Adam, S.; Polini, M. Gate-tunable coherent perfect absorption of terahertz radiation in graphene. $2 D$ Mater. 2014, 1, 031001.

(27) Fan, Y. C.; Zhang, F. L.; Zhao, Q.; Wei, Z. Y.; Li, H. Q. Tunable terahertz coherent perfect absorption in a monolayer graphene. Opt. Lett. 2014, 39, 6269-6272.

(28) Li, S. C.; Duan, Q.; Li, S.; Yin, Q.; Lu, W. X.; Li, L.; Gu, B. M.; Hou, B.; Wen, W. J. Perfect electromagnetic absorption at one-atomthick scale. Appl. Phys. Lett. 2015, 107, 181112.

(29) Pirruccio, G.; Moreno, L. M.; Lozano, G.; Rivas, J. G. Coherent and Broadband Enhanced Optical Absorption in Graphene. ACS Nano 2013, 7, 4810-4817.

(30) Rao, S. M.; Heitz, J. J. F.; Roger, T.; Westerberg, N.; Faccio, D. Coherent control of light interaction with graphene. Opt. Lett. 2014, $39,5345-5347$

(31) Sensale-Rodriguez, B.; Yan, R. S.; Liu, L.; Jena, D.; Xing, H. G. Graphene for Reconfigurable Terahertz Optoelectronics. Proc. IEEE 2013, 101, 1705-1716.

(32) Kakenov, N.; Balci, O.; Polat, E. O.; Altan, H.; Kocabas, C. Broadband terahertz modulators using self-gated graphene capacitors. J. Opt. Soc. Am. B 2015, 32, 2548-2548.

(33) Brida, D.; Tomadin, A.; Manzoni, C.; Kim, Y. J.; Lombardo, A.; Milana, S.; Nair, R. R.; Novoselov, K. S.; Ferrari, A. C.; Cerullo, G.; Polini, M. Ultrafast collinear scattering and carrier multiplication in graphene. Nat. Commun. 2013, 4, 1987.

(34) Shi, S. F.; Tang, T. T.; Zeng, B.; Ju, L.; Zhou, Q.; Zettl, A.; Wang, F. Controlling Graphene Ultrafast Hot Carrier Response from Metal-like to Semiconductor-like by Electrostatic Gating. Nano Lett. 2014, 14, 1578-1582.

(35) Frenzel, A. J.; Lui, C. H.; Shin, Y. C.; Kong, J.; Gedik, N. Semiconducting-to-Metallic Photoconductivity Crossover and Temperature-Dependent Drude Weight in Graphene. Phys. Rev. Lett. 2014, 113, 056602.

(36) Adam, S.; Hwang, E. H.; Galitski, V. M.; Das Sarma, S. A selfconsistent theory for graphene transport. Proc. Natl. Acad. Sci. U. S. A. 2007, 104, 18392-18397. 